\title{
On standard extensions of local fields
}

\begin{abstract}
Akram Lbekkouri
BP: 10507, Casa-Bandoeng Casablanca, 20002 - Morocco

E-mail: lbeka11@gmail.com

Abstract

Let $L / K$ be any separable extension of complete discrete valued fields of degree $p$. This work, is a study of some "standard over-extensions" of $L / K$, with the description of their Galois groups. The second target, which is the aim of this work, concerns the Galois closure of $L / K$. The study of the normal case has been done in some former work.
\end{abstract}

2010 Mathematics Subject Classification. 11S15.

Keywords. Wild ramification, Discriminant, Eisenstein polynomials, Standard extensions of a local field, Intermediate extension, Semi-direct product.

\section{Introduction}

Let $L / K$ be a separable extension of degree $p$ of complete discrete valued fields having residue fields of characteristic $p>0$. The content of this paper is as follows:

Section 1 is a general view of the standard over-extensions of $K$. Some specific results and examples on the extension $M=K\left(\left(K^{\star}\right)^{1 / p-1}\right) / K$, in general, are also given.

Section 2 is a description of the Galois groups of the standard extensions, the question of the finitude of the Number of Galois extensions having a given degree is studied and a Method for the determination of some cyclic extensions of a local number field is given.

Section 3 is the study of the Galois closure of $L / K$ (the aim of this work). The existence of the intermediate extension and an explicit determination of it are studied.

\section{Standard over-extensions}

By "local field" we mean a complete discrete valued field, meanwhile "standard over-extensions" of a local field $K$ are, the maximal abelian extension $M$ of $K$ of exponent $p-1$, the maximal $p$-abelian extension of $M$, and the Galois closure of a $p$-extension of $K$.

\subsection{Case of finite residue field}

Let $K$ a local field with finite residue field, $k=\mathbb{F}_{p^{f}}$. The maximal abelian extension of exponent $p-1$ of $K$ is $M=K\left(\left(K^{\star}\right)^{1 / p-1}\right)$, regardless of the characteristic of $K$, that is the compositum of two cyclic Kummer linearly disjoint extensions of $K$ both of degree $p-1$. The unramified and a totally ramified $K(\sqrt[p-1]{\pi})$ ( $\pi$ uniformizer of $K) . M / K$ is the compositum of all cyclic extensions of $K$ of degree dividing $p-1$. From Kummer Theory for abelian extensions (see [12] $\operatorname{ch}:$ VI), $\Gamma=\operatorname{gal}(M / K)$ 
(the Galois group of $M / K)$ is dual to $K^{\star} / K^{\star(p-1)}$, under the pairing:

$$
\begin{aligned}
\varphi: \quad \Gamma \times\left(K^{\star} / K^{\star(p-1)}\right) & \longmapsto \\
(\sigma, \bar{x}) & \longmapsto \sigma(y) / y
\end{aligned}
$$

so $\mathbb{F}_{p}^{\star} \subset K^{\star}$, is identified with the group of the $p-1$-th roots of unity. $N$ the maximal abelian extension of exponent $p$ of $M$ is compositum of all extensions of $K$ of degree $p$.

First $\underline{\text { case }} \operatorname{char}(K)=0$

Here, $N=M\left(\sqrt[p]{M^{\star}}\right)$; furthermore $M / K$, and $N / M$ are normal.

- $\Gamma=\operatorname{gal}(M / K)$, is abelian of degree $(p-1)^{2}$ isomorphic to $(\mathbb{Z} /(p-1) \mathbb{Z})^{2}$.

- Write $\Delta=\operatorname{gal}(N / M)$ seen as $\Gamma$-module (from the action of $\Gamma$ on it, $\Gamma$ acts on $M^{\star} / M^{\star p}$ and on $\mu_{p} \subset M . \Delta \simeq \operatorname{Hom}\left(M^{\star} / M^{\star p},<\zeta_{p}>\right)$ so it is isomorphic to the filtered $\Gamma$-module $M^{\star} / M^{\star p}$ of $\mathbb{F}_{p}$-dimension $p^{2+\left[M: \mathbb{Q}_{p}\right]}$. See Remark (1.1).

- $\mathcal{G}=\operatorname{gal}(N / K)$, need not be nilpotent. It is a semidirect product $\mathcal{G}=\Delta \rtimes \Gamma_{0}$, where $\Gamma_{0}$ is a subgroup of $\mathcal{G}$ isomorphic to $\Gamma$ ( Schur-Zassenhaus Theorem, see [14]Chap.7. Th.7.24).

Remark 1.1. If the extension $L / \mathbb{Q}_{p}$ is finite then the order of the group $L^{\star} / L^{\star p}$ is

- 1. If $L$ contains the $p$-th roots of unity then the order of the group $L^{\star} / L^{\star p}$ is $p^{2+\left[L: \mathbb{Q}_{p}\right]}$.

- 2. If $L$ does not contain the $p$-th roots of unity $p^{1+\left[L: \mathbb{Q}_{p}\right]}$

Set $\left[L: \mathbb{Q}_{p}\right]=e f$, from $L^{\star}=\pi^{\mathbb{Z}} \times \mu_{p^{f}-1} \times \mathbb{U}_{1}$ for $\pi$ a uniformizer of $L, \mu_{n}$ the group of the $n$-th roots of unity and $\mathbb{U}_{1}$ the group $U_{1}=\left\{a \in L ; a-1 \in \mathcal{M}_{L}\right\}$, so $L^{\star} \simeq \mathbb{Z} \times \mu_{p^{f}-1} \times \mathbb{U}_{1}$. From Prop. 10 , Ch.XIV $\S .4$ in [17], $\mathbb{U}_{1}$ is a direct product of a cyclic $p$-group and a $\mathbb{Z}_{p}$-module of rank $\left[L: \mathbb{Q}_{p}\right]$, so $\mathbb{U}_{1} \simeq \mu_{p^{h}} \times \mathbb{Z}_{p}^{\left[L: \mathbb{Q}_{p}\right]}$ with $h \geq 0, \mu_{p^{h}} \subset L$ and $\mu_{p^{h+1}}$ not in $L$, so $h=0$ if and only if $L$ does not contain $\mu_{p}$ (see the following Note). So,

$$
\begin{gathered}
L^{\star} \simeq \mathbb{Z} \times \mu_{p^{f}-1} \times \mu_{p^{h}} \times \mathbb{Z}_{p}^{\left[L: \mathbb{Q}_{p}\right]} \\
L^{\star} / L^{\star p} \simeq \mathbb{Z} / p \mathbb{Z} \times\{1\} \times \mu_{p^{h}} / \mu_{p^{h}}^{p} \times(\mathbb{Z} / p \mathbb{Z})^{\left[L: \mathbb{Q}_{p}\right]}
\end{gathered}
$$

- If $h=0$ then $\mu_{p^{h}} / \mu_{p^{h}}^{p}$ is of dimension zero.

- If $h>0$ then $\mu_{p^{h}} / \mu_{p^{h}}^{p} \simeq \mathbb{Z} / p \mathbb{Z}$ that is of dimension 1 .

In consequence $\operatorname{dim}\left(L^{\star} / L^{\star p}\right)=1+1+\left[L: \mathbb{Q}_{p}\right]$ if $h>0$ meanwhile $\operatorname{dim}\left(L^{\star} / L^{\star p}\right)$

$=1+\left[L: \mathbb{Q}_{p}\right]$ if $h=0$. See for example Corollary of Proposition $6 \S .3$ Ch.II in [7].

Note: we prove, $\mathbb{U}_{1} \simeq \mu_{p^{h}} \times \mathbb{Z}_{p}^{\left[L: \mathbb{Q}_{p}\right]}$.

For $L / \mathbb{Q}_{p}$ finite, the $p$-adic logarithm is a $\mathbb{Z}_{p}$-module homomorphism $\log : \mathbb{U}_{1} \rightarrow \mathcal{M}_{L}$, and $\operatorname{ker}(\log )$ is the $p$-th power roots of unity in $L$. This kernel is finite, since high $p$-th power order roots of unity have high degree over $\mathbb{Q}_{p}$, and can't lie in a finite extension of $\mathbb{Q}_{p}$ if the order is sufficiently large. The $p$-adic logarithm is an isomorphism from a sufficiently small closed disc $\mathcal{D}$ around 1 to a sufficiently small closed disc around 0 , with its inverse being the $p$-adic exponential. A closed disc around 0 in $\mathcal{M}_{L}$ is a scalar multiple of $\mathcal{M}_{L}$, and $\mathcal{M}_{L} \simeq \mathbb{Z}_{p}^{\left[L: \mathbb{Q}_{p}\right]}$, so $\mathcal{D} \simeq \mathbb{Z}_{p}^{\left[L: \mathbb{Q}_{p}\right]}$. Since $\mathcal{D}$ is a $\mathbb{Z}_{p}$-submodule of $\mathbb{U}_{1}$ with finite index, $\mathbb{U}_{1}$ is a finitely generated (multiplicative) $\mathbb{Z}_{p}$-module that contains a submodule of finite index which is free of $\operatorname{rank}\left[L: \mathbb{Q}_{p}\right]$, so by the structure theorem for 
finitely generated modules of a PID, $\mathbb{U}_{1}$ as a $\mathbb{Z}_{p}$-module is $\mathbb{T} \times \mathbb{Z}_{p}^{\left[L: \mathbb{Q}_{p}\right]}, \mathbb{T}$ is the torsion submodule of $\mathbb{U}_{1}$. The submodule $\mathbb{T}$ is $\mathbb{T}=\mu_{p^{h}} \subset \mathbb{U}_{1}$. Thus $\mathbb{U}_{1} \simeq \mu_{p^{h}} \times \mathbb{Z}_{p}^{\left[L: \mathbb{Q}_{p}\right]}$. A special case is for $p=2$, since all 2-adic field contains the 2-th roots of unity nevertheless the result still holds. For example, if $L=\mathbb{Q}_{2}, \mathbb{Q}_{2}^{\star} \simeq \mathbb{Z} \times \mathbb{Z}_{2} \times \mathbb{Z} / 2 \mathbb{Z} \simeq \mathbb{Z} \times \mathbb{U},\left(\mathbb{U}=\mathbb{U}_{1}\right)$, and $\mathbb{U}_{1}=\mathbb{Z}_{2}^{\star}=\{+/-1\} \times\left(1+4 \mathbb{Z}_{2}\right) \simeq\{+/-1\} \times \mathbb{Z}_{2}$ since the 2 -adic logarithm is an isomorphism between $1+4 \mathbb{Z}_{2}$ and $4 \mathbb{Z}_{2} \simeq \mathbb{Z}_{2}$.

Remark 1.2. Since $N / M$ a $p$-elementary abelian, $\operatorname{gal}(N / M)=\Delta \simeq(\mathbb{Z} / p \mathbb{Z})^{n}$ with $n=2+\left[M: \mathbb{Q}_{p}\right]$ and from classical group theory $(\mathbb{Z} / p \mathbb{Z})^{n}$ has exactly

$$
\left(\begin{array}{c}
n \\
i
\end{array}\right)_{p}=\frac{\left(p^{n}-1\right)\left(p^{n-1}-1\right) \ldots\left(p^{n-i+1}-1\right)}{\left(p^{i}-1\right)\left(p^{i-1}-1\right) \ldots(p-1)}
$$

subgroups of order $p^{i},\left(\left(\begin{array}{c}n \\ i\end{array}\right)_{p}\right.$ the Gaussian $p$-binomial coefficient $(n \text { choose } i)_{p}$ for $\left.i \leq n\right)$. (The number of $i$-dimensional subspaces of an $n$-dimensional vector space over $\mathbb{F}_{p}$ ). By the theorem of classical Galois theory, $N / M$ contains $\left(\begin{array}{c}n \\ i\end{array}\right)_{p}$ extensions of $M$ of degree $p^{n-i}$.

$\underline{\text { Second }} \underline{\text { case: }} \operatorname{char}(K)=p>0, K=F((T))$, with $F$ a finite field. Then $N=M\left(\wp^{-1}(M)\right)$; $\left(\wp: x \rightarrow x^{p}-x\right)$ (Artin-Schreier).

- $\Gamma=\operatorname{gal}(M / K)$, which is abelian of degree $(p-1)^{2}$ isomorphic to $(\mathbb{Z} /(p-1) \mathbb{Z})^{2}$.

- $\Delta=\operatorname{gal}(N / M)$ is isomorphic to the filtered $\Gamma$-module $M /(\wp(M))$ of $\mathbb{F}_{p}$-dimension $+\infty$, which is abelian too of exponent $p$, isomorphic to a countably infinite product of copies of $\mathbb{Z} / p \mathbb{Z}$ in general see Proposition (1.5).

- $\mathcal{G}=\operatorname{gal}(N / K), \mathcal{G}$ need not be nilpotent, since $\mathcal{G}=\Delta \rtimes \Gamma_{0}, \Gamma \simeq \Gamma_{0} \subset \mathcal{G}$, (Generalized SchurZassenhaus [13]. §.2.3; page: 41)). Indeed from Krull topology, (see [12] ch:VII), $\Delta$ is a closed normal subgroup of $\mathcal{G}$ and the exponents are relatively prime. So, we have a split short exact sequence $1 \rightarrow \Delta \rightarrow \mathcal{G} \rightarrow \Gamma_{0} \rightarrow 1$.

Note: Having $\Gamma \simeq \Gamma_{0}$, in the next, we write $\Gamma$ instead of $\Gamma_{0}$ since no confusion can occur.

Remark 1.3. $\Delta$ is the single Sylow $p$-subgroup of $\mathcal{G}$, so the number of subgroups of $\mathcal{G}$ of order $p^{i}$ equals the number of subgroups of $\Delta$ of order $p^{i}$ for all $i$, namely,

$$
\left(\begin{array}{c}
n \\
i
\end{array}\right)_{p}=\frac{\left(p^{n}-1\right)\left(p^{n-1}-1\right) \ldots\left(p^{n-i+1}-1\right)}{\left(p^{i}-1\right)\left(p^{i-1}-1\right) \ldots(p-1)}
$$

\subsection{On the prime and Equi-characteristic Case}

Remain that for a complete discrete valued field $K$ having the same characteristic $p$ as its residue field $F$ we can write $K=F((T))$ with $T$ a transcendental element over $F$.

\subsubsection{Infinitude of $K / \wp(K)$}

Proposition 1.4. $K=F((T))$, with $F$ a complete discrete valued field of characteristic $p$ then $K / \wp(K)$, is countably infinite, $\left(\wp: x \rightarrow x^{p}-x\right)$.

Proof. Consider $\frac{1}{T^{n}}$, for $n>0$ and $p$ does not divide $n$. If $\frac{1}{T^{n}}-\frac{1}{T^{n^{\prime}}} \in \wp(K)$, with $n \neq n^{\prime}$ and $p$ does not divide $n n^{\prime}$, then $\frac{1}{T^{n}}-\frac{1}{T^{n^{\prime}}}=f^{p}-f$, for some $f \in K=F((T))$ but $f \notin K=F[[T]]$, 
necessarily (since $n, n^{\prime}>0$ and distinct ) (which is no more true if $F$ is finite). Thus $f$ has a leading polar term with degree $-r<0$, so $f^{p}$ has a pole with degree $-r p<-r$, that is $f^{p}-f$ has a pole of order $r p$ that is divisible by $p$ yet the difference $\frac{1}{T^{n}}-\frac{1}{T^{n^{\prime}}}$, does not have this property since $n$ and $n^{\prime}$ are distinct and not divisible by $p$. So, we found infinitely many different elements outside of a subspace.

For the infinity of the codimension. $\left(T^{n}\right)_{n}$ with $n$ negative prime to $p$ numbers is free in $K / \wp(K)$. Let $n_{1}<\cdots<n_{m}$ be negative prime to $p$ integers, and $a_{1}, \ldots, a_{m} \in F$ non-zero. We have to prove that $f=a_{1} T^{n_{1}}+\cdots+a_{m} T^{n_{m}}$ does not lie in $\wp(K)$. Let $v$ be the canonical valuation of $K=F((T))$. Then $v(f)=n_{1}<0$. By contradiction, suppose that $f=g^{p}-g$ for some $g \in K$. Then $v(g)<0$, so $v\left(g^{p}-g\right)=p v(g)$. $f=g^{p}-g$ implies that $n_{1}=v(f)=v\left(g^{p}-g\right)=p v(g)$, thus $p$ divides $n_{1}$. So, we get the contradiction. Now, by Hensel's Lemma $\wp(K)$ contains an open neighborhood of 0 so $K / \wp(K)$ is just countably infinite.

Q.E.D.

Note: Prop.(1.4) can be generalized to any infinite and commutative field $K$, $\operatorname{char}(K)=p$ with $\wp(K) \varsubsetneqq K$ (strict inclusion). Indeed, the equality can occur, for example if $K$ is algebraically closed, the equation $T^{p}-T-t$ is separable, with $K$ separably closed and $\operatorname{char}(K)=p$ we get $\wp(K)=K, K / \wp(K)$ is then trivial.

Let $K$ be a commutative and infinite field and $L / K$ finite with $[L: K]>1$. The element 1 can be extended to a $K$-basis $e_{1}, \ldots, e_{n}$ of $L$, with $e_{1}=1$ and $n>1$. Then $L=K e_{1}+K e_{2}+\ldots+K e_{n}=$ $K+K e_{2}+\ldots+K e_{n}$ (the sums are direct sums). Passing to additive quotient groups, $L / K$ is isomorphic to $K e_{2}+\ldots+K e_{n}$, which is infinite since $K$ is infinite. So, a similar argument works when $L$ is any field extension of $K$ that is larger than $K$ (not just finite extensions of $K$ ) by using a $K$-basis of $L$ that contains $K$.

\subsubsection{Description of the product $\Delta$}

Proposition 1.5. For $L=\mathbb{F}((T))$ a local functional field with $\mathbb{F}$ a finite field of characteristic $p$, let $N$ be the maximal exponent- $p$ abelian extension of $L$. Then $\operatorname{gal}(N / L)$ is a product of an countable infinite product of copies of $\mathbb{Z} / p \mathbb{Z}$.

Proof. By Kummer's theory, gal $(N / L)$ embeds into $\operatorname{Hom}(L / \wp(L), \mathbb{Z} / p \mathbb{Z})$ $\simeq(\mathbb{Z} / p \mathbb{Z})^{(\alpha)}$, and is a direct product of a non-necessarily countable number of copies of $\mathbb{Z} / p \mathbb{Z}$, of course $L / \wp(L) \simeq \operatorname{gal}(N / L)$ and $L / \wp(L)$ embeds into $(\mathbb{Z} / p \mathbb{Z})^{(\alpha)}$. Since $L / \wp(L)$ is just countably infinite (see Prop.1.4) and thus has only countably infinite dimension then with Pontryagin duality that swaps direct sums for direct products we see that $\operatorname{gal}(N / L)$ is thereby obtained as a countably infinite product.

Q.E.D.

By use of the notations of $\S .1 .2 . K=\mathbb{F}((T)$ ) ( $\mathbb{F}$ finite of characteristic $p$ ), $M / K$ is Kummerabelian of degree $(p-1)^{2}$, then $M=K\left(\sqrt[p-1]{K^{\star}}\right)$ with $M=V((X))$ too $(V$ finite $) V=\mathbb{F}(\sqrt[p-1]{(\varepsilon)})(\varepsilon$ a generator of $\mathbb{F}^{\star}$, and $\left.X=\sqrt[p-1]{T}\right)$. Now, by "continuity of roots" for separable monic polynomials, 
there are only countably many finite separable extensions of a local function fields see Example(2.9) (as such fields have a countable dense subset), $\Delta$ is necessarily a countable infinite product of copies of $\mathbb{Z} / p \mathbb{Z}$. Furthermore, Prop. (1.5) gives a direct proof of

Corollary 1.6. From Prop.(1.5). The group $\Delta=\operatorname{gal}(N / M)$ (where $N=M\left(\wp^{-1}(M)\right)$ and $M=$ $\left.K\left(\sqrt[p-1]{K^{\star}}\right)\right)$, is a product of an countable infinite product of copies of $\mathbb{Z} / p \mathbb{Z}$.

\subsection{Remarks on the extension $M=K\left(\left(K^{\star}\right)^{1 / p-1}\right) / K$ in general}

- In local case with finite residue field of characteristic $p$ we have seen that

$M=K\left(\left(K^{\star}\right)^{1 / p-1}\right) / K$, is an abelian extension of degree $(p-1)^{2}$ the Galois group of which is isomorphic to $(\mathbb{Z} /(p-1) \mathbb{Z})^{2}$.

- Meanwhile, if $\mathrm{K}$ is a complete field with respect to a discrete valuation having a residue field not necessarily finite of characteristic $p$, then we have $M=K\left(\left(K^{\star}\right)^{1 / p-1}\right) / K$ is not necessarily finite, but it is still abelian of exponent $p-1$, since $K$ contains the $p-1$-th roots of unity.

- Otherwise, the extension $M / K$ need not be finite; if it is finite it need not be Galois; and if it is finite and Galois it need not have that Galois group. Indeed see the following.

Example 1.7. 1). • Let $K=k((t))$, where $k=\mathbb{Q}\left(\zeta_{3}\right)$ and $\zeta_{3}$ is a primitive cube root of unity. So $K$ is a complete discretely valued field.

Let $p=3 . k\left(\left(k^{\star}\right)^{1 / p-1}\right) / k$ is infinite. Hence so is $K\left(\left(K^{\star}\right)^{1 / p-1}\right) / K$.

2). $\quad K=\mathbb{Q}\left(\zeta_{3}\right)$ where $\zeta_{3}$ is a 3 -th root of unity. Therefore, $M / K=K\left(\left(K^{\star}\right)^{1 / p-1}\right) / K=$ $\mathbb{Q}\left(\zeta_{3}\right)\left(\left(\mathbb{Q}\left(\zeta_{3}\right)^{\star}\right)^{1 / 2}\right) / \mathbb{Q}\left(\zeta_{3}\right)$, is infinite, since adjoining to $K$ the square roots of different prime elements of $\mathbb{Z}\left[\zeta_{3}\right]$ will lead to disjoint quadratic extensions whose composite has degree a large power of 2 (the power being the number of primes).

\section{More generally we have the following result:}

3). - "Consider $K=\mathbb{Q}\left(\zeta_{p}\right)$ where $\zeta_{p}$ is a $p$-th root of unity, $p$ being an odd prime number. Then $K\left(\sqrt[1 / p-1]{K^{\star}}\right) / K=\mathbb{Q}\left(\zeta_{p}\right)\left(\sqrt[1 / p-1]{\mathbb{Q}\left(\zeta_{p}\right)^{\star}}\right) / \mathbb{Q}\left(\zeta_{p}\right)$, is infinite".

Indeed, from the well known result "For relatively prime integers $a_{1}, \ldots, a_{n}$, the $2^{n}$ algebraic numbers $\sqrt{a_{i_{1}}, \ldots, a_{i_{k}}}$ with $i_{1}<\ldots<i_{k}$ and $0 \leq k \leq n$ are linearly independent over $\mathbb{Q}$, so are a $\mathbb{Q}$-basis for $\mathbb{Q}\left(\sqrt{a_{i_{1}}}, \ldots, \sqrt{a_{i_{k}}}\right)$. In particular, the degree of that field over $\mathbb{Q}$ is the maximum possible $2^{n}$ ", we can deduce that $\mathbb{Q}\left(\left(\mathbb{Q}^{\star}\right)^{1 / 2}\right) / \mathbb{Q}$ is infinite. Since $\mathbb{Q}\left(\zeta_{p}\right) / \mathbb{Q}$ is finite then $Q\left(\zeta_{p}\right)\left(\left(Q\left(\zeta_{p}\right)^{\star}\right)^{1 / 2}\right) / \mathbb{Q}\left(\zeta_{p}\right)$ is infinite, therefore $\mathbb{Q}\left(\zeta_{p}\right)\left(\left(\mathbb{Q}\left(\zeta_{p}\right)^{\star}\right)^{1 / p-1}\right) / \mathbb{Q}\left(\zeta_{p}\right)$ is infinite too. The result is proved.

Note that the degree of $\mathbb{Q}\left(\zeta_{p}\right)\left(\sqrt{a_{i_{1}}}, \ldots, \sqrt{a_{i_{k}}}\right)$ over $\mathbb{Q}\left(\zeta_{p}\right)$ is $2^{n}$ or $2^{n-1}$; it depends on whether the set the numbers $a_{i}$ union $+p$ or $-p$ is still independent or not and $\sqrt{+p}$ or $\sqrt{-p}$ belongs to $\mathbb{Q}\left(\zeta_{p}\right)$, depends on whether $p \equiv 1 \bmod 4$ or $p \equiv 3 \bmod 4$.

$4)$. - Let $k$ be an algebraically closed field of characteristic 0 , and let $K=k((t))$.

Then $K\left(\left(K^{\star}\right)^{1 / p-1}\right) / K$ is Galois with group $\mathbb{Z} /(p-1) \mathbb{Z}$, not $(\mathbb{Z} /(p-1) \mathbb{Z})^{2}$.

$5)$. - Let $\mathrm{k}$ be the field of 3 elements, and let $K=k((t))$.

Let $p=11$. Then $K\left(\left(K^{\star}\right)^{1 / p-1}\right) / K$ is Galois with group $\mathbb{Z} / 2 \mathbb{Z} \times \mathbb{Z} / 10 \mathbb{Z}$. 
6). - Let $k$ be the field of 3 elements, and let $K=k((t))$.

Let $p=7$ Then $K\left(\left(K^{\star}\right)^{1 / p-1}\right) / K$ has degree 12 (not 36), but it is not Galois because it is not separable, since $t^{1 / 3}$ is in this field.

7). • "For any fractions field $K$, with characteristic $p \neq 2$, of a Dedkind ring $\mathfrak{A}$ having infinite many prime ideals, we have $M=K\left(\left(K^{\star}\right)^{1 / p-1}\right) / K$, is infinite".

Indeed, it suffices to notice that when adjoining to $K$ the square roots of two different prime elements of $\mathfrak{A}$ will lead to disjoint quadratic extensions. In fact, let $L=K(\sqrt{p})$ and $L^{\prime}=K(\sqrt{q})$. They are both quadratic. Necessarily $L \cap L^{\prime}=K$ otherwise $L=L^{\prime}$, this means that $\sqrt{q}=a+b \sqrt{p}$ for $a, b \in K$, thus $q=a^{2}+2 a b \sqrt{p}+b^{2} p$. Clearly $b$ has to be non-zero. If $a$ is also non-zero, then this formula shows $\sqrt{p} \in K$, so $a$ has to be zero. Then $q=b^{2} p$, localizing at $q, p$ is a unit and $q$ is a uniformizer so this cannot happen.

8). • In contrary, in characteristic $2 \mathbb{F}_{2}(T)(\sqrt{T})=\mathbb{F}_{2}(T)(\sqrt{T+1})$ Is a counter-example.

\section{Note:}

Concerning items 7) and 8), the different result for characteristic 2 is really just an artifact. More generally, if $p$ is any prime and a positive integer $n$ is not a power of $p$, then $M=K\left(\left(K^{\star 1 / n}\right) / K\right.$ is infinite for rings as in item 7). Of course if $p$ is prime and $n=p-1$, then $n$ cannot be a power of a prime $q$ unless $q=2$, which leads to the item 8). But if we take a different $n$ (e.g. take $n=p-2$ ), then characteristic 2 need not be the exception.

\section{Description of the over-extensions}

\subsection{Case of mixed characteristic}

\subsubsection{Explicit description of the semidirect product}

From $\S .1 .1$ First case, $\Gamma \simeq(\mathbb{Z} /(p-1) \mathbb{Z})^{2}$, and $\Delta \simeq(\mathbb{Z} / p \mathbb{Z})^{n}$. Write $\Delta=<\alpha_{1}, \alpha_{2}, \ldots, \alpha_{n}>$. $M^{\star} / M^{\star p}$ being a $\mathbb{F}_{p}[\Gamma]$-module of dimension $n$, by local class field theory $M^{\star} / M^{\star p} \simeq \Delta=\operatorname{gal}(N / M)$. Furthermore, $\Delta \simeq \operatorname{Hom}\left(M^{\star} / M^{\star p},\langle\zeta>)\right.$ with $\zeta$ a primitive $p$-th root of unity. So, $N$ is generated over $M$ by $n$ elements $b_{i}$ such that $b_{i}^{p} \in M$ that is $N=M\left(b_{1}, b_{2}, \ldots, b_{n}\right)$, so consider $\Delta=<$ $\alpha_{1}, \alpha_{2}, \ldots, \alpha_{n}>$ such that $\alpha_{i}\left(b_{i}\right)=\zeta_{i} b_{i}$ with $\zeta_{i}$ a $p$-th root of unity, and $\alpha_{i}\left(b_{j}\right)=b_{j}$ if $i \neq j$. To sum up we have the result:

Proposition 2.1. For $N=M\left(b_{1}, b_{2}, \ldots, b_{n}\right)$, with $b_{i}^{p} \in M$. Then $\Delta=\operatorname{gal}(N / M)=<\alpha_{1}, \alpha_{2}, \ldots, \alpha_{n}>$ is defined by $\alpha_{i}\left(b_{i}\right)=\zeta_{i} b_{i}$ with $\alpha_{i}\left(b_{j}\right)=b_{j}$ if $i \neq j$.

Let $\varphi:(\mathbb{Z} /(p-1) \mathbb{Z})^{2} \rightarrow \operatorname{Aut}\left((\mathbb{Z} / p \mathbb{Z})^{n}\right)$ a non trivial homomorphism. Set $\Delta \rtimes_{\varphi} \Gamma=(\mathbb{Z} / p \mathbb{Z})^{n} \rtimes_{\varphi}(\mathbb{Z} /(p-1) \mathbb{Z})^{2}=<\alpha_{1}, \alpha_{2}, \ldots, \alpha_{n}>\rtimes_{\varphi}<g_{1}, g_{2}>$, by use of the basic representation theory, every representation of $\Gamma$ is completely reducible by the theorem of Maschke see [4]. Further $\left|\operatorname{Hom}\left(\Gamma, \mathbb{F}_{p}^{\star}\right)\right|=|\Gamma|$, so every irreducible representation of $\Gamma$ over $\mathbb{F}_{p}$ has dimension 1. then, if $V$ is a vector space over $\mathbb{F}_{p}$ and $\varphi: \Gamma \rightarrow A u t\left(V_{\mathbb{F}_{p}}\right)$ a homomorphism, there exists a basis 
$B$ of $V$ and homomorphisms $\varphi_{b}: \Gamma \rightarrow \mathbb{F}_{p}^{\star}, b \in B$ such that $\varphi(g)(b)=\varphi_{b}(g) b$ for every $g \in \Gamma$ and every $b \in B$. So we get:

Proposition 2.2. The semi-direct product $\mathcal{G}$, $\mathcal{G}=\Delta \rtimes_{\varphi} \Gamma=(\mathbb{Z} / p \mathbb{Z})^{n} \rtimes_{\varphi}(\mathbb{Z} /(p-1) \mathbb{Z})^{2}=<\alpha_{1}, \alpha_{2}, \ldots, \alpha_{n}>\rtimes_{\varphi}<\sigma, \tau>$, is defined by the $2 n$ relations: $\sigma \alpha_{i} \sigma^{-1}=\zeta_{i} \alpha_{i}$, and $\tau \alpha_{i} \tau^{-1}=\xi_{i} \alpha_{i}$, for $i=1, \ldots, n ; \zeta_{i}, \xi_{i}$ being elements of $(\mathbb{Z} / p \mathbb{Z})^{\star}$.

That is by terms of characters, for $\chi_{i} \in \hat{\Gamma}=\operatorname{Hom}\left(\Gamma, \mathbb{F}_{p}^{\star}\right.$ ) (dual of $\Gamma$ ); write $M_{1}=\operatorname{diag}\left(\chi_{1}(\sigma), \chi_{2}(\sigma), \ldots, \chi_{n}(\sigma)\right)$, and $M_{2}=\operatorname{diag}\left(\chi_{1}(\tau), \chi_{2}(\tau), \ldots, \chi_{n}(\tau)\right)$, for the diagonal matrices images of $\sigma$ and $\tau$, then the action above becomes: $\sigma \alpha_{i} \sigma^{-1}=\chi_{i}(\sigma) \alpha_{i}$, and $\tau \alpha_{i} \tau^{-1}=\chi_{i}(\tau) \alpha_{i}$.

\subsubsection{Noticeable remarks on the group $\mathcal{G}$}

\section{Remark 2.3. :}

- 1. In general such groups are metabelian, but nonnilpotent. Meanwhile, they can be nilpotent, then abelian, if and only if for all $i ; \zeta_{i}=\xi_{i}=1$ ( $\mathcal{G}$ is then a direct product).

Concerning the center $Z(\mathcal{G})$ of $\mathcal{G}$. Since $(\mathbb{Z} / p \mathbb{Z})^{n}$ and $(\mathbb{Z} /(p-1) \mathbb{Z})^{2}$ are abelian, any generator of the first subgroup that commutes with the generators of the second lies in the center and vis versa. So:

- 2. $\mathcal{G}=\Delta \rtimes_{\varphi} \Gamma=(\mathbb{Z} / p \mathbb{Z})^{n} \rtimes_{\varphi}(\mathbb{Z} /(p-1) \mathbb{Z})$ note the action of $\Gamma$ on $\Delta$ the homomorphism $\varphi: \Gamma \rightarrow \operatorname{Aut}(\Delta)$ then $\operatorname{ker}(\varphi)$ consists of all $\sigma^{a} \tau^{b}$ for which $\zeta_{i}^{a} \xi_{i}^{b}=1$ for all $i$. Put $C=C_{\Delta}(\Gamma)=$ $C_{\Delta}(\sigma) \cap C_{\Delta}(\tau)$ it is described in terms of the $i$ such that $\zeta_{i}=\xi_{i}=1$. Then $C=\Delta \cap Z(\mathcal{G})$. For $\sigma \tau \in \mathcal{G}$ with $\sigma \in \Delta$ and $\tau \in \Gamma$ then $\sigma \tau \in C_{\mathcal{G}}(\Gamma) \Leftrightarrow \sigma \in C$. On the other hand $\sigma \tau \in C_{\mathcal{G}}(\Delta) \Leftrightarrow \tau \in$ $C_{\Gamma}(\Delta)=\operatorname{ker}(\varphi)$. Finally $Z(\mathcal{G})=C_{\mathcal{G}}(\Gamma) \cap C_{\mathcal{G}}(\Delta)$.

- 4. For $m<n$ if there are exactly $m$ indices $i$ with $\zeta_{i}=\xi_{i}=1$ then $\# Z(\mathcal{G}) \geq p^{m}$.

- 5. $\# Z(\mathcal{G})>p^{m}$ if and only if there exist $a, b$ not both are zero, such that $0 \leq a, b<p-1$, and $\zeta_{i}^{a} \cdot \xi_{i}^{b}=1$ for all $i$. Indeed, for $g \in \mathcal{G}, g=n h$ with $n \in(\mathbb{Z} / p \mathbb{Z})^{n}$ and $h \in(\mathbb{Z} /(p-1) \mathbb{Z})^{2}, g$ is central if and only if both $n$ and $h$ are central. Since, central elements in $\mathcal{G}$ contained in $(\mathbb{Z} / p \mathbb{Z})^{n}$ are generated by the $\alpha_{i}$ for which $\zeta_{i}=\xi_{i}=1$. So $h=\sigma^{a} \tau^{b}$ is central if and only if the condition above holds, so there can be more than $p^{m}$ elements in the center. Also, $\# Z(Z(\mathcal{G}))=p^{m} \cdot c$ with $c$ a proper divisor of $(p-1)^{2}$.

- 6. Particularly if $\zeta_{i}=\xi_{i}$ for all $i$; then $\sigma^{-1} \tau$ lies in the center that is $(p-1) \mid \# Z(\mathcal{G})$. Likewise if $\zeta_{i}=\xi_{i}^{-1}$ for all $i$, then $\tau \sigma$ lies in the center that is $(p-1) \mid \# Z(\mathcal{G})$ too.

- 7. If none of the conditions 4.), 5.) and 6.) hold then $\mathcal{G}$ is centerless.

Proposition 2.4. Let $G_{0}$ be a subgroup of $\mathcal{G}=(\mathbb{Z} / p \mathbb{Z})^{n} \rtimes_{\varphi}(\mathbb{Z} /(p-1) \mathbb{Z})^{2}$ of index $p$, then $G_{0} \cap(\mathbb{Z} / p \mathbb{Z})^{n}$ is normal in $\mathcal{G}$.

Proof. First note that $(\mathbb{Z} / p \mathbb{Z})^{n}$, is the $p$-Sylow subgroup of $\mathcal{G}$ and is normal in it. Since $G_{0}$ contains a copy of $(\mathbb{Z} /(p-1) \mathbb{Z})^{2}$, then $(\mathbb{Z} /(p-1) \mathbb{Z})^{2}$, normalizes $G_{0}$ and therefore normalizes 
$G_{0} \cap(\mathbb{Z} / p \mathbb{Z})^{n}$. By other hand $(\mathbb{Z} / p \mathbb{Z})^{n}$ normalizes $G_{0} \cap(\mathbb{Z} / p \mathbb{Z})^{n}$, since $(\mathbb{Z} / p \mathbb{Z})^{n}$ is abelian. In consequence $G_{0} \cap(\mathbb{Z} / p \mathbb{Z})^{n}$ is normal in $\mathcal{G}$.

Q.E.D.

Remark 2.5. The result above does not mean that any subgroup of index $p$ of $(\mathbb{Z} / p \mathbb{Z})^{n}$, is normal in $\mathcal{G}=(\mathbb{Z} / p \mathbb{Z})^{n} \rtimes_{\varphi}(\mathbb{Z} /(p-1) \mathbb{Z})^{2}$. See the following counter-examples.

Example 2.6. (Counter-example)

In Proposition (2.4) when considering $p=3, n=2$ take for example for the action defining the semi-direct product $[\varphi(x, y)](a, b)=(a, y b)$ (here we identified $\mathbb{Z} /(p-1) \mathbb{Z}$ with $\left.\mathbb{F}_{p}^{\star}\right)$. The subgroup $\left\{(a, a) \mid a \in \mathbb{Z}_{3}\right\}$ is obviously not normal in $\mathcal{G}$.

Example 2.7. (Counter-example)

Let $K=\mathbb{Q}_{3}$, consider $M=K\left(\sqrt{K^{\star}}\right)=\mathbb{Q}_{3}(i, \sqrt{3})$, and consider $E=M(\sqrt[3]{1+\sqrt{3}})$, that is a normal 3-extension of $M$. The Galois closure of $E / K$ is $N=M\left(\sqrt[3]{M^{\star}}\right)$ i.e., $N=M(\sqrt[3]{1+\sqrt{3}}, \sqrt[3]{1-\sqrt{3}})$ and $\operatorname{gal}(N / M)=(\mathbb{Z} / 3 \mathbb{Z})^{2}$. But $E / K$ is not normal otherwise there should be an intermediate subextension $E^{\prime} / K$ of degree 3 of $E / K$ and an automorphism $\sigma$ of $E$ that maps $\sqrt{3}$ to $-\sqrt{3}$, which is the identity on $E^{\prime}$, furthermore $\sigma(\sqrt[3]{1+\sqrt{3}})$, must be a cubic root of $\sigma(1+\sqrt{3})=1-\sqrt{3}$, but $E$ contains no such root, since $E$ is strictly contains in $N$. Hence the subgroup $g a l(N / E)$ is not normal in $\operatorname{gal}(N / K)$.

\subsection{Equi-characteristic Case}

For $\Delta$ a $p$-profinite group, product of a countable number of copies of $\mathbb{Z} / p \mathbb{Z}, N$ is generated over $M$ by a countable number of elements $b_{i}$ such that $b_{i}^{p}-b_{i} \in M$. So, $\Delta$ is generated by $\alpha_{i}$ of order $p$ with $\alpha_{i}^{j}\left(b_{i}\right)=b_{i}+j$ for $0 \leq j<p, i \in N$ and $\alpha_{i}\left(b_{k}\right)=b_{k}$ for $i \neq k$. So:

Proposition 2.8. With a countable number of relations, we define $\mathcal{G}=\Delta \rtimes_{\varphi} \Gamma=<\alpha_{1}, \alpha_{2}, \ldots, \alpha_{n}, \ldots>$ $\rtimes_{\varphi}<\sigma, \tau>$, is $\sigma \alpha_{i} \sigma^{-1}=\zeta_{i} \alpha_{i}$, and $\tau \alpha_{i} \tau^{-1}=\xi_{i} \alpha_{i}$; for $i \in N$; and $\zeta_{i}, \xi_{i} \in(\mathbb{Z} / p \mathbb{Z})^{\star}$. That are, $\sigma \alpha_{i} \sigma^{-1}=\chi_{i}(\sigma) \alpha_{i}$, and $\tau \alpha_{i} \tau^{-1}=\chi_{i}(\tau) \alpha_{i}$ where $\chi_{i} \in \hat{\Gamma}$.

\subsection{On the Number of Galois extensions having a given degree}

The finitude of the number of all extensions of a local number field having a given degree" was studied and explicitly computed first by I.R.Safarevič in [15], M.Krasner in [6] then by J.P.Serre in [16]. In characteristic $p>0$ this result holds no more. See the Example:

Example 2.9. For instance,

- The field $\mathbb{F}_{p}((X)),\left(\mathbb{F}_{p}\right.$ of $p$ elements), has only one inseparable extension of degree $p$. Indeed for $L$ an inseparable extension of degree $p, L^{p}=\mathbb{F}_{p}((X))$, of course $p$-th power in $K=\mathbb{F}_{p}((X))$ are Laurent series in $X^{p}\left(\mathbb{F}_{p}\right.$ is perfect). So, if $f \in K, f=a_{0}+a_{1} X++a_{p-1} X^{p-1}$ each $a_{i}$ is a $p$-th power. $K(\sqrt[p]{f})$ lies in $K(\sqrt[p]{X})$, and so $K(\sqrt[p]{X})$ is the only purely inseparable extension of degree $p$, and $\left.L=\mathbb{F}_{p}\left((X)^{1 / p}\right)\right)$. Meanwhile, it has infinitely many separable ones (Artin-Schreier) of this 
degree. In fact the question reduces to whether, $K / \wp(K),\left(\wp: x \rightarrow x^{p}-x\right)$, is infinite? which is true. Prop. ( 1.4).

- In imperfect residue field case, we have the following beautiful example. $K=k(x)((z))(k$ is algebraically closed of characteristic $p$ ) has infinitely many extensions of degree $p$. Extensions given by $y^{p}-y=x^{j},(j \in \mathbb{N}$ and $j \nmid p)$, are all disjoint Galois $p$-extensions.

Now, let us first state some important results on groups:

Lemma 2.10. A finitely generated group $G$ has only finitely many normal subgroups of a given index $n$, and only finitely many subgroups of $G$ of bounded index.

ProOF. Let $G=<x_{1}, \ldots, x_{k}>$ be a finitely generated group and $H$ a fixed finite group. There are finitely many homomorphisms from $G$ to $H$ (for each tuple $g_{1}, \ldots, g_{k}$ there is at most one sending $x_{i}$ to $g_{i}$ ). So there are finitely many normal subgroups $N$ of $G$ such that $G / N \simeq H$ (for each such $N$ there exists at least one homomorphism from $G$ to $H$ with kernel $N$ ). As, up to isomorphism there are finitely many groups of fixed order, then there are finitely many normal subgroup of $G$ having fixed (or even bounded index). Let $K$ be a subgroup of $G$ of finite index $m$, it has at most $m$ conjugates $K_{1}, \ldots, K_{l}$ and the intersection of all $K_{i}$ is a normal subgroup of index at most $m^{l} \leq m^{m}$. (The normal core of $K$ ). As for a normal subgroup $N$ of index $s$ there are at most $2^{s}$ subgroups containing $N$, then the number of subgroups of bounded index in $G$ is bounded.

Q.E.D.

Theorem 2.11. Let $G$ be a topologically finitely generated profinite group, then:

- For each natural number $n$ the number of open subgroups of $G$ of index $n$ is finite.

- Identity element 1 of $G$ has a fundamental system of neighborhoods consisting of countable chain of open characteristic subgroups of $G=V_{0} \supseteq V_{1} \supseteq V_{2} \ldots$ See [13] (Prop. 2.5.1)

The Galois group of any infinite extension is a profinite group, the converse is also true. So in case of Theorem (2.11), "the finitude" still holds.

Corollary 2.12. If $\operatorname{gal}\left(K^{s} / K\right)$ is topologically finitely generated, then there are only finitely many Galois extensions of a given degree of $K$. Particularly if $K$ is quasi-finite.

In "Serre's sense" a field is said to be quasi-finite if it is perfect and $\operatorname{gal}\left(K^{s} / K\right) \simeq \widehat{\mathbb{Z}}$.

\subsection{Method for the determination of some cyclic extensions of a local number field}

Let $K / \mathbb{Q}_{p}$ be a finite extension, $\left[K: \mathbb{Q}_{p}\right]=r$. Set $K_{c}$ the compositum of all cyclic extensions of $K$ of degree $p$.

\subsubsection{On the compositum of all cyclic $p$-extensions}

Proposition 2.13. With the hypothesis above,

$1 \bullet\left[K_{c}: K\right]=p^{r+1}$ and $\operatorname{gal}\left(K_{c} / K\right) \simeq(\mathbb{Z} / p \mathbb{Z})^{r+1}$, if the $p-t h$ roots of unity are in $K .2$ $\left[K_{c}: K\right]=p^{r+2}$ and $\operatorname{gal}\left(K_{c} / K\right) \simeq(\mathbb{Z} / p \mathbb{Z})^{r+2}$, if $K$ contains the $p-t h$ roots of unity. 
Proof. By local class field theory, $K^{\star} / K^{\star p}$ is isomorphic to the Galois group of the maximal elementary abelian $p$-extension of $K$ ie. $K_{c}$. Remark.(1.1 ) gives the result.

Q.E.D.

\subsubsection{Explicitness for the case $K=\mathbb{Q}_{p}$}

\section{Application: The Maximal $p$-abelian extension of $\mathbb{Q}_{p}$}

For $p \neq 2, \mathbb{Q}_{p}$ has exactly $p+1$ cyclic extensions of degree $p$, all are totally ramified except one is unramified. For $p=2$ a detailed classification of the quadratic and the quartic extensions is given in [10]. Put $r=1$ in Prop.(2.13) to determine the compositum of all cyclic extensions of $\mathbb{Q}_{p}$ of degree $p$. Exhibit two cyclic linearly disjoint extensions of degree $p$ of $\mathbb{Q}_{p}$ ( the unramified $\mathbb{Q}_{p}(\lambda)$, and the subextension $\mathbb{Q}_{p}(\eta)$ (totally ramified) of degree $p$ of $\mathbb{Q}_{p}\left(\zeta_{p^{2}}\right) ; \zeta_{p^{2}}$ is a primitive $p^{2}$-th root of unity). The $p+1$ cyclic extensions of degree $p$ of $\mathbb{Q}_{p}$ are the subextensions of $\mathbb{Q}_{p}(\lambda, \eta)$. Respectively write, $G_{\lambda}=\operatorname{gal}\left(\mathbb{Q}_{p}(\lambda)\right) / \mathbb{Q}_{p}$ and $G_{\eta}=\operatorname{gal}\left(\mathbb{Q}_{p}(\eta)\right) / \mathbb{Q}_{p}$. There are natural isomorphisms from $G_{\lambda}$ and $G_{\eta}$ into $\mathbb{F}_{p}$.

To determine the primitive elements, set $\eta=1+\sum_{0<i<p^{2} ; i^{p-1} \equiv 1 \bmod p} \zeta_{p^{2}}^{i}$ an uniformizer (the trace), their conjugates $\eta_{k}=1+\sum_{0<i<p^{2} ; i^{p-1} \equiv 1 \bmod p} \zeta_{p^{2}}^{i+k p}$, with $0 \leq k \leq p-1$ (action of $\mathbb{F}_{p}$ on the conjugates of $\eta$ ). For a prime $q, q \equiv 1 \bmod p$; and $p^{(q-1) / p}$ not congruent to $1 \bmod q$ and $p^{(q-1) / p}$ not congruent to $1 \bmod q$, write $\lambda=\sum_{j \bmod q ; j^{(q-1) / p} \equiv 1 \bmod q} \zeta_{q}^{j}$ the conjugates are $\lambda_{k}=\sum_{j \bmod q ; j^{(q-1) / p} \equiv 1 \bmod q} \zeta_{q}^{j} \zeta_{p}^{k}$, with $0 \leq k \leq p-1$. The expression $\lambda_{r_{1}} \eta_{s_{1}}+\ldots+\lambda_{r_{p}} \eta_{s_{p}}$ gives the primitive elements for the $p$-cyclic extensions of $\mathbb{Q}_{p}$.

Example 2.14. For a numerical example, consider the case $p=7$ we have $\left[\mathbb{Q}_{7}\left(\zeta_{49}\right): \mathbb{Q}_{7}\right]=42$, so we can take $\eta=1+\zeta_{49}+\zeta_{49}^{-1}+\zeta_{49}^{18}+\zeta_{49}^{-18}+\zeta_{49}^{19}+\zeta_{49}^{-19}$ thus we get $\left[\mathbb{Q}_{7}(\eta)\right.$ : $\left.\mathbb{Q}_{7}\right]=7$ with $\mathbb{Q}_{7}(\eta) / \mathbb{Q}_{7}$ cyclic totally ramified. Then by taking $q=29$ we get $\left[\mathbb{Q}_{7}\left(\zeta_{29}\right): \mathbb{Q}_{7}\right]=28$ therefore, we can take $\lambda=\zeta_{29}+\zeta_{29}^{-1}+\zeta_{29}^{12}+\zeta_{29}^{-12}$ and thus $\left[\mathbb{Q}_{7}(\lambda): \mathbb{Q}_{7}\right]=7$ with $\mathbb{Q}_{7}(\lambda) / \mathbb{Q}_{7}$ cyclic unramified.

For a detailed study (see [8] $§ .3$ page 139). With software Pari, for several values of $p$, the Eisenstein polynomials corresponding to the $p$ cyclic extensions are determined, as well as their reduites (in Krasner's sense).

\subsubsection{Determination of the cyclic extensions of degree $d$ of $\mathbb{Q}_{p}$, with $d \mid p-1$ )}

$p$ an odd prime, and $d=q_{1}^{r_{1}} \cdot q_{2}^{r_{2}} \ldots q_{s}^{r_{s}}$ ( $q_{i}$ prime) for $d \mid p-1$. By Kummer theory, the cyclic extensions of degree $d$ of $\mathbb{Q}_{p}$ are in bijection with the cyclic subgroups of order $d$ of $\mathbb{Q}_{p}^{\star} / \mathbb{Q}_{p}^{\star}$. Since $\mathbb{Q}_{p}^{\star}=p^{Z} \times Z_{p}=p^{Z} \times \mu_{p-1} \times U_{1}\left(\mu_{n} n\right.$-th roots of unity), and $U_{1}^{d}=U_{1}$, so $\mathbb{Q}_{p}^{\star} / \mathbb{Q}_{p}^{\star d} \simeq$ $p^{Z} / p^{d Z} \times \mu_{p-1} / \mu_{(p-1) / d} \simeq<p>\times<\zeta>$ a product of two cyclic groups of order $d$. These extensions come from taking a $d$-th root of $\xi p^{i},(i$ integer determined $\bmod d, \xi$ is a $p-1$-th root of unity (determined up to multiplication by a $((p-1) / d)$-th root of unity). This gives the product of two cyclic groups of order $d$. Now The number of cyclic non-isomorphic extensions of degree $d$ of $\mathbb{Q}_{p}$ is equal to the number of cyclic subgroups of order $d$ of $(\mathbb{Z} / d \mathbb{Z}) \times(\mathbb{Z} / d \mathbb{Z})$. Since, a cyclic group of order $d$ contains $\varphi(d)$ elements of order $d$, (Euler's totient). For $g(d)$ the number of elements 
of order $d$ in a group, the number of cyclic subgroups is $g(d) / \varphi(d)$. The order of any element of $G$ (direct product of two cyclic groups of order $d$ ) divides $d$. If $m$ divides $d$, then the set of elements whose orders divide $m$ is the subgroup of $G$ which is the direct product of two cyclic groups of order $m$, whose order is $m^{2}$. So, if $g(m)$ is the number of elements of order exactly $m, m^{2}=\sum_{k \mid m} g(k)$, and by möbius inversion $(\mu) g(m)=\sum_{k \mid m} k^{2} \mu(m / k)$. For $m=d$ gives the number of elements of order $d$ in $G$. The number of cyclic subgroups of order $d$ in the group $G$ is $g(d) / \varphi(d)=\left(\sum_{k \mid d} k^{2} \mu(d / k)\right) / \varphi(d)$.

For $d=60, \varphi(d)=16$ and then the number of elements of order 60 is $60^{2}-30^{2}-20^{2}-12^{2}+10^{2}+$ $6^{2}+4^{2}-2^{2}=2304$, so the number of cyclic subgroups of order 60 is 144 .

For $d$ a prime, it is $\left(d^{2}-1\right) /(d-1)=d+1$ see Huppert in [3] (Hilfssatz 8.5). The number of cyclic groups of order $d$ in an elementary abelian $d$-group of rank $n$, is $\left(d^{n}-1\right) /(d-1)$.

Description of Galois groups of cyclic extensions of degree $d$ of $\mathbb{Q}_{p}$ with $d \mid p-1$. For $r=1$ and $s=1$ then $d$ is prime, these are in bijection with the pairs $(i, j) \in(\mathbb{Z} / d \mathbb{Z})^{2}$ with either $i=1$ or $(i, j)=(0,1)$, corresponding to the $\mathbb{F}_{d}$ points on the projective line.

A similar description for prime-powers, say $q^{r}$, the subgroups generated by pairs $(1, j)$ for all $j$ and those generated by pairs $(i, 1)$ for all $i$ divisible by the prime $q$.

For the general case use the canonic splitting into the direct product of the Sylow subgroups and combine for each Sylow subgroup.

Example 2.15. Description of cyclic extensions of degree 3 of $\mathbb{Q}_{7}$ ?

By local class field theory, this is the same as the number of one-dimensional subspaces of the $\mathbb{F}_{3}$-vector space $\mathbb{Q}_{7}^{\star} /\left(\mathbb{Q}_{7}^{\star}\right)^{3}$. As 3 divides $6=7-1$, this is 2 -dimensional: the cubes in $\mathbb{Q}_{7}^{\star}$ are $7^{3 n} \varepsilon$ where $\varepsilon=+-1(\bmod 7)$. So there are 4 such extensions.

$\mathbb{Q}_{7}$ contains the cube roots of unity. So, the degree 3 cyclic extensions are Kummer extensions, they are generated by the cube roots of $2,7,14$ and 28 .

\section{Embedding of an extension of prime degree in its Galois closure}

\subsection{Existence of the intermediate extension}

Proposition 3.1. Let $K$ be a commutative field, for every separable extension $L / K$ of degree $p$, $p$ an odd prime, $G=\operatorname{gal}\left(L_{C}\right) / K$ the Galois group of the Galois closure of $L / K$ is solvable. Then there exists a cyclic extension $F / K$ of degree $m$ dividing $p-1$ such that $L F / F$ is cyclic of degree $p$ and $L F / K$ is Galois (ie. $\left.L_{C}=L F\right)$. Furthermore if $L / K$ is not cyclic $(L F / K$ is hence not abelian), then $L$ has exactly $p$ conjugates over $K$ in $L F$.

Proof. $G$ is solvable, its order is divisible by $p$ but not by $p^{2}$. Seen as a transitive subgroup of the symmetric group $\mathfrak{S}_{p}$, then according to ([1], ch.3, th.7) $G$ contains a unique subgroup $P$ of order $p$ so it is normal in $G . P$ is contained in its normalizer $N(P)$ in $\mathfrak{S}_{p}$. Also $N(P)$ seen as the affine linear group $G A_{1}\left(\mathbb{F}_{p}\right)$, we have the isomorphism $\mathbb{F}_{p}^{\star} \rightarrow A u t(P)$, and a split short exact 
sequence $: 1 \rightarrow P \rightarrow N(P) \rightarrow \mathbb{F}_{p}^{\star} \rightarrow 1$

Furthermore, $N(P)$ is isomorphic to the group of all $2 \times 2$ matrices over $G F(p)$ of the form $\left(\begin{array}{cc}x & 0 \\ y & 1\end{array}\right)$ In consequence $G / P$ is cyclic of order $m$ dividing $p-1$. Therefore, and since $G \subset N(P)$ it is also a semidirect product $G=P \rtimes M$ with $M$ cyclic of order $m$.

If the semidirect product is a direct product then it is cyclic since $m$ and $p$ are co-prime.

Otherwise $G$ is not abelian. In such case $M$ being cyclic then all its conjugates are cyclic too. Write $m$ in the form $m=\prod_{i=1}^{r} m_{i}^{\alpha_{i}}, m_{i}$ being different prime numbers, and $N$ for the number of the conjugates of $M$ (note that according to Hall's theorem(see [14]Chap5. Th5.23. page 85) all the subgroups of $G$ of order $m$ are conjugate). Since $M$ is cyclic it contains one and only one subgroup $M_{i}$ of order $m_{i}^{\alpha_{i}}$ (Sylow $m_{i}$-subgroup of $G$ ) which is cyclic too. Conversely every Sylow $m_{i}$-subgroup of $G$ can be embedded in some conjugate of $M$. So the number $N$ must divide $m p$, being $N \equiv 1$ modulo $m_{i}$ for all $i$, thus $(N, m)=1$. So the number of conjugates of $M$ is exactly $p$ if $G$ is not cyclic. Set $F$ the field fixed by $P$, then the Galois closure of $L / K$ is $L_{C}=L F$. The proof is ended.

Q.E.D.

Remark 3.2. $F$ is unique. Now, $L / K$ being of prime degree, from now on we can suppose that $L / K$ is totally ramified (so $L F / F$ is too) and write $L F=F(\pi)$.

\subsection{Intermediate extension, explicit determination}

\section{From now on, assume that $K$ has a finite residue field of characteristic $p$.}

\subsubsection{Description of the Galois closure}

Recall that the compact group $\Gamma \simeq \operatorname{Hom}\left(K^{\star} / K^{\star p-1}, \mu_{p-1}\right)$ then by duality $\Gamma \simeq K^{\star} / K^{\star p-1}$. Hence $\Gamma$ is of the exponent $p-1$, and $M / K$ is Kummer abelian relatively to $p-1$. The subextension $F$ of $L_{C} / K$ (Prop. 3.1), and of $M / K$, is cyclic Kummer of degree $m$ dividing $p-1$ then, $F=K(\sqrt[m]{b})$, with $b \in K^{\star}$. So, $K(\sqrt[m]{b})=K(\sqrt[m]{d})$ if and only if there exists an integer $k \geq 1$; with $(k, m)=1$ such that $d \in b^{k} K^{\star m}$.

By considering the quotient group $K^{\star} / K^{\star m}$ the order of the class $b K^{\star m}$; in it is $m$. Since $m$ is dividing $(p-1), K^{\star} / K^{\star m} \simeq(\mathbb{Z} / m \mathbb{Z}) \times(\mathbb{Z} / m \mathbb{Z})$; therefore $K^{\star} / K^{\star m}$ is of order $m^{2}$. The number of the distinct Kummer cyclic extensions of $K$ of degree $m$ is exactly the number of cyclic subgroups of order $m$ in $\left(K^{\star} / K^{\star m}\right)$. So, the number of the cyclic distinct Kummer extensions of $K$ of degree $m$ equals the number of the cyclic subgroups of order $m$ included in $(\mathbb{Z} / m \mathbb{Z}) \times(\mathbb{Z} / m \mathbb{Z})$, so by writing $m=p_{1}^{\alpha_{1}} \ldots p_{r}^{\alpha_{r}}$, we get this number equals to $\left(p_{1}^{\alpha_{1}}+p_{1}^{\alpha_{1}-1}\right) \ldots\left(p_{r}^{\alpha_{r}}+p_{r}^{\alpha_{r}-1}\right)$. Furthermore $\operatorname{gal}(F / K) \simeq H ; H$ being a subgroup of $\operatorname{gal}\left(L_{C} / K\right)$ and $L_{C}$ the Galois closure of $L / K$; is a cyclic group of order $m$ dividing $(p-1)$ that can be embedded in $\mu_{p-1}$ the group of the $p-1$ th roots of unity. So, Schur-Zassenhaus theorem ( [14]Chap.7. Th.7.24., page:151) ensures the semi direct product $\operatorname{gal}\left(L_{C} / K\right) \simeq \operatorname{gal}\left(L_{C} / F\right) \rtimes H$. From local class field theory see [2] the 
isomorphism between the three groups $\operatorname{gal}(F / K) \simeq H \simeq K^{\star} / N_{F / K}\left(F^{\star}\right)$ of order $m$, and the surjective homomorphism $s: K^{\star} / K^{\star m} \simeq(\mathbb{Z} / m \mathbb{Z}) \times(\mathbb{Z} / m \mathbb{Z}) \mapsto K^{\star} / N_{F / K}\left(F^{\star}\right)$.

\subsubsection{The group $\operatorname{gal}(L F / K)$}

Since $\operatorname{gal}(F / K)$ is cyclic of order $m$ dividing $p-1$, write $\operatorname{gal}(F / K)=<\varepsilon>$ with $\varepsilon(\sqrt[m]{b})=\xi_{m}(\sqrt[m]{b})$, where $\xi_{m}$ a primitive $m$-th root of unity and name the extension of $\varepsilon$ to $F(\pi), \varepsilon$ too. Since $\operatorname{gal}(F(\pi) / F)$ is cyclic of order $p$ write $\operatorname{gal}(F(\pi) / F)=\langle\sigma\rangle$. $L F / K$ being Galois, consider $\tau$ any element of $\operatorname{gal}(L F / K)$, thus $\tau=\sigma^{i} \varepsilon^{j}$, with $1 \leq i \leq p$ and $1 \leq j \leq m$, then from the normality of $<\sigma>$ in $\operatorname{gal}(L F / K)$, we have the identity

$$
\tau \sigma \tau^{-1}=\sigma^{t} \text { with } 1 \leq t \leq p-1
$$

Consider the affine group $A G L(1, p)$, of all maps from $\mathbb{F}_{p}$ to itself in the form $x \mapsto u x+v$ where $u \neq 0$ in $\mathbb{F}_{p} . \operatorname{gal}(L F / K)$ has order $m p$ and is isomorphic to a subgroup of $A G L(1, p)$, which is isomorphic to the subgroup $G L_{2}(\mathbb{Z} / p \mathbb{Z})$, of the matrices in form $\left(\begin{array}{ll}u & v \\ 0 & 1\end{array}\right)$ an automorphism $\delta$ corresponds to $\left(\begin{array}{cc}u & v \\ 0 & 1\end{array}\right) ; \delta\left(\xi_{p}\right)=\xi_{p}^{u}$, and $\delta(x)=\xi_{p}^{v} x ; \xi_{p}$, is a primitive $p$-th root of unity. Pick a generator $g$ of $(\mathbb{Z} / p \mathbb{Z})^{\star}$, for a generator of $g a l(F / K)$ take, $\varepsilon: x \mapsto g x$ that corresponds to $\left(\begin{array}{ll}g & 0 \\ 0 & 1\end{array}\right)$ and for a generator $\sigma$ of $\operatorname{gal}(L F / F), \sigma: x \mapsto x+1$ that corresponds to $\left(\begin{array}{ll}1 & 1 \\ 1 & 0\end{array}\right)$ then $\varepsilon \sigma \varepsilon^{-1}=\sigma^{g}$. For any $\tau$ of $\operatorname{gal}(L F / K) ; \tau=\sigma^{i} \varepsilon^{j}$, with $1 \leq i \leq p$ and $1 \leq j \leq m, \tau \sigma \tau^{-1}=\sigma^{g^{j}}$, also $g$ must verify $g^{m}=1$ in $\mathbb{F}_{p} .(\mathbb{Z} / p \mathbb{Z})^{\star}$, has $\varphi(m)$ elements of order $m, \varphi($.) (Euler's totient). Meanwhile the equation $x^{m}=1 \bmod p$ has exactly $m$ solutions in $(\mathbb{Z} / p \mathbb{Z})^{\star},(m$ divides $p-1$ which is the order of $\left.(\mathbb{Z} / p \mathbb{Z})^{\star}\right)$, these solutions are the elements of the cyclic subgroup of order $m$ of the cyclic group $(\mathbb{Z} / p \mathbb{Z})^{\star}$, and is isomorphic to the group of the $m$-th roots of unity.

\subsection{Generation of the intermediate extension}

\subsubsection{Ramification elements of $L F / K$ :}

$L F=F(\pi), \pi$ uniformizer of $L$ and of $L F$ too. $d_{(.)}, e_{(.)}$and $f_{(.)}$the respective discriminant, ramification index and residual degree. So $e_{L F / F}=e_{L / K}=p ; f_{L F / F}=f_{L / K}=1$.

Write $e_{F / K}=e_{L F / L}=t=\#\left|G_{0} / G_{1}\right|$ and $f_{F / K}=f_{L F / L}=r$ that is the order of $G / G_{1}$ (with respectively $G$ the Galois $G_{0}$ the inertia and $G_{1}$ the ramification groups).

For any $K$-homomorphism $\sigma$ of $L$, define the break relative to $\sigma$ as $v=v_{L}\left(\frac{\sigma(\pi)}{\pi}-1\right) . v$ is independent of $\pi$ and $\sigma$ and depends of $L / K$ only, see [5]. With a prime degree it is unique with $v \leq \frac{e p}{p-1}$. Its integrity is a necessary condition for the normality of $L / K$.

By computing $v_{K}\left(d_{L F / K}\right)$ in two different ways, along the towers $L F / F / K$ and $L F / L / K$ we get $v_{F}\left(d_{L F / F}\right)=(p-1)(1+v)$; furthermore we have $v_{K}\left(d_{F / K}\right)=v_{L}\left(d_{L F / L}\right)=(t-1) r=m-r$. 
In conclusion we get $v_{K}\left(d_{L / K}\right)=(p-1)\left(1+\frac{v}{t}\right)$. So,

$$
\operatorname{gcd}(v, t)=1 .
$$

\subsubsection{Explicit computation of the break}

Let $f(X)=\sum_{i=0}^{p} a_{i} X^{i}$, be an Eisenstein polynomial of degree $p a_{i} \in K(f(\pi)=0)$, write $\pi=$ $\pi_{1}, \pi_{2}, \ldots, \pi_{p}$, for the roots of $f(X)=0$. Set $f_{0}(X)=X^{-1} f(\pi(X+1))=X^{-1} \sum_{i=0}^{p} a_{i} \pi^{i}(X+1)^{i}=$ $X^{-1} \sum_{i=0}^{p} \sum_{t=0}^{i} a_{i} \pi^{i}\left(\begin{array}{c}i \\ t\end{array}\right) X^{t}=\sum_{j=0}^{p-1} \sum_{i=j+1}^{p}\left(\begin{array}{c}i \\ j+1\end{array}\right) a_{i} \pi^{i} X^{j}=$ $\sum_{j=0}^{p-1} d_{j} X^{j}$, with $d_{j}=\sum_{i=j+1}^{p}\left(\begin{array}{c}i \\ j+1\end{array}\right) a_{i} \pi^{i}, \quad d_{p-1}=\pi^{p}$, and $d_{0}=\sum_{i=1}^{p}\left(\begin{array}{l}i \\ 1\end{array}\right) a_{i} \pi^{i}=\sum_{i=1}^{p} i a_{i} \pi^{i}$. Then $w=\frac{v_{L}\left(d_{0}\right)-v_{L}\left(d_{p-1}\right)}{p-1}$, so $v_{L}\left(d_{0}\right)=(p-1) w+p\left(v_{L}(\right.$.$\left.) normalized valuation of L\right)$.

Since $v_{L}\left(d_{0}\right)=\inf _{1 \leq t \leq p}\left(v_{L}\left(t a_{t}\right)+t\right)$, there exists $a_{k}$ the principal coefficient of $f$, such that $w=\frac{v_{L}(k)+v_{L}\left(a_{k}\right)+k-p}{p-1}$. Having $d_{0} \equiv k a_{k} \pi^{k}$, modulo $\pi^{v_{L}\left(k a_{k}\right)+k+1}$, two cases can be distinguished. First case, $k \neq p$, and then $w=\frac{v_{L}\left(k a_{k}\right)+k-p}{p-1}$, with $k=(p-1) w-v_{L}\left(a_{k}\right)+p$, in the Second $k=p$ (necessarily $\operatorname{char}(K)=0$ ) so $w=\frac{p e}{p-1}$. With $w=\frac{v}{t}$ we have:

$$
d_{0} \equiv\left(k a_{k} \pi^{-v_{L}\left(a_{k}\right)}\right)\left(\pi^{(p-1) w+p}\right) \operatorname{modulo} \pi^{(p-1) w+p+1} .
$$

$\left(k a_{k} \pi^{-v_{L}\left(a_{k}\right)}\right.$ being an unit of $\left.L\right)$.

\subsubsection{Explicit computation of the primitive element}

Consider $g(X)=X^{-1} f(\pi+X)=\sum_{t=0}^{p-1} b_{t} X^{t}$, its roots are $\theta_{i}=\sigma^{i}(\pi)-\pi$, for $1 \leq i \leq p-1$. $\left(\sigma^{i}(\theta) \equiv \theta \bmod \pi\right.$, so, $\left.N_{L F / F}(\theta) \equiv \theta^{p} \equiv \theta \bmod \pi\right)$, then $L\left(\theta_{2}, \ldots, \theta_{p}\right)$ is the splitting field of $f$ over $K . g(X)=\sum_{t=0}^{p-1} \sum_{i=t+1}^{p}\left(\begin{array}{c}i \\ t+1\end{array}\right) a_{i} \pi^{i-t-1} X^{t} ; b_{t}=\sum_{i=t+1}^{p}\left(\begin{array}{c}i \\ t+1\end{array}\right) a_{i} \pi^{i-t-1}, b_{p-1}=1$ and $\prod_{i=1}^{p-1} \theta_{i}=b_{0}=\sum_{i=1}^{p}\left(\begin{array}{l}i \\ 1\end{array}\right) a_{i} \pi^{i-1}=\sum_{i=1}^{p} i a_{i} \pi^{i-1}$, so $d_{0}=b_{0} \pi$.

$v_{L}\left(b_{0}\right)=\inf _{1<t<p}\left(v\left(t a_{t}\right)+t-1\right)=v\left(d_{0}\right)-1=(p-1)(w+1)$. So, $v_{L}\left(a_{k}\right)=(p-1) w-k+p$. Then $\prod_{i=1}^{p-1} \theta_{i}=b_{0} \equiv k a_{k} \pi^{k-1}=\left(k a_{k} \pi^{-v_{L}\left(a_{k}\right)}\right)\left(\pi^{(p-1)(w+1)}\right)$ modulo $\pi^{(p-1) w+p}$.

Write $\gamma=-b_{0}=-k a_{k} \pi^{k-1}$, and extend the normalized valuation $v_{L}($.$) of L$ to $L F$ in a nonnormalized way $\left(v_{L F}(\pi)=1\right)$. Denote by $g_{1}(X)=X^{p-1}-\gamma\left(\right.$ its roots are the $\zeta_{p-1}^{i} \sqrt[p-1]{\gamma}$, where $\zeta_{p-1}$ is a $(p-1)$-th root of unity), and by $\theta^{\prime}$ any root of $g_{1}(X)=0$. Compute the expression $g\left(\theta^{\prime}\right)-g_{1}\left(\theta^{\prime}\right)=g\left(\theta^{\prime}\right)$ in two different ways:

$$
\begin{aligned}
g\left(\theta^{\prime}\right)-g_{1}\left(\theta^{\prime}\right) & =\theta^{p-1}-\theta^{p-1}+\sum_{i=1}^{p-2} b_{i} \theta^{i}+\sum_{i=1}^{p} i a_{i} \pi^{i-1}+\gamma \\
& =\sum_{i=1}^{p-2} b_{i} \theta^{\prime i}+\sum_{i=1, i \neq k}^{p} i a_{i} \pi^{i-1} .
\end{aligned}
$$

All valuations in the sums are $\geq(p-1) w+p$. Since $g\left(\theta^{\prime}\right)=\prod_{i=1}^{p-1}\left(\theta^{\prime}-\theta_{i}\right)$ then $v_{L F}\left(\prod_{i=1}^{p-1}\left(\theta^{\prime}-\theta_{i}\right)\right)=$ $\sum_{i=1}^{p-1} v_{L F}\left(\theta^{\prime}-\theta_{i}\right) \geq(p-1) w+p=(p-1)(w+1)+1$, so there exists $i_{0}$ with $v_{L F}\left(\theta^{\prime}-\theta_{i_{0}}\right) \geq$ $(w+1)+\frac{1}{p-1}$, that is $v_{L F}\left(\theta^{\prime}-\theta_{i_{0}}\right)>(w+1)$, by Krasner's Lemma (see [9]) $L\left(\theta^{\prime}\right)=L\left(\theta_{i_{0}}\right)=$ $L(\sqrt[p-1]{\gamma})=L\left(\theta_{2}, \ldots, \theta_{p}\right)=K(\pi, \sqrt[p-1]{\gamma})=L F$. Then:

Theorem 3.3. With the current notations, let $L / K$ be a separable extension of degree $p$. If there exist an index $k, 1 \leq k \leq p-1$, such that $v_{L}\left(a_{k}\right)+k=\inf _{1 \leq i \leq p}\left(v_{L}\left(a_{i}\right)+i\right)$, then 
$K\left(\sqrt[p-1]{-k a_{k} \pi^{k-1}}\right) / K$ is cyclic Kummer extension of degree $m, m$ dividing $p-1$. Furthermore, the splitting field of $f$ over $K$ is $K\left(\pi, \sqrt[p-1]{-k a_{k} \pi^{k-1}}\right)$.

Notice that $\theta^{\prime} \equiv \theta_{i_{0}} \equiv \theta \bmod \pi$ and take $\theta^{\prime}=\sqrt[p-1]{\gamma}$, then

$$
\theta^{\prime}=\sqrt[p-1]{\gamma} \equiv \theta \text { modulo } \pi
$$

Furthermore, from the equality $k-1=(p-1)(w+1)-v_{L}\left(a_{k}\right)$, and since $w=\frac{v}{t}$ :

$$
\theta^{\prime}=\sqrt[p-1]{\gamma}=\zeta_{p-1} \sqrt[p-1]{-k a_{k} \pi^{-v_{L}\left(a_{k}\right)}} \pi^{\frac{v}{t}+1}
$$

$(p-1)$ being prime to $p$ then $L^{\star} / L^{\star(p-1)} \simeq K^{\star} / K^{\star(p-1)} \simeq \mathbb{Z} /(p-1) \mathbb{Z} \times \mathbb{Z} /(p-1) \mathbb{Z}$, so

$$
\begin{array}{lrr}
L^{\star} / L^{\star(p-1)} & \rightarrow & K^{\star} / K^{\star(p-1)} \\
\delta L^{\star(p-1)} & \rightarrow & N_{L / K}(\delta) K^{\star(p-1)},
\end{array}
$$

is an isomorphism. Since $N_{L / K}\left(\frac{\gamma}{N_{L / K}(\gamma)}\right) \in K^{\star(p-1)}$, thus the pre-image $\frac{\gamma}{N_{L / K}(\gamma)} \in L^{\star(p-1)}$, that is $\sqrt[p-1]{\frac{\gamma}{N_{L / K}(\gamma)}} \in L^{\star}$. So $L(\sqrt[p-1]{\gamma})=K(\pi, \sqrt[p-1]{\gamma})=K\left(\pi, \sqrt[p-1]{N_{L / K}(\gamma)}\right)$, and then $F=$ $K\left(\sqrt[p-1]{N_{L / K}(\gamma)}\right)$, and $L F=K\left(\pi, \sqrt[p-1]{N_{L / K}(\gamma)}\right)$. By other words we can take

$$
\sqrt[p-1]{N_{L / K}(\gamma)} \text { as primitive element of } F / K
$$

If the principal coefficient is $a_{p}=1(\operatorname{char}(K)=0), L F=L(\sqrt[p-1]{-p} \pi)=L(\sqrt[p-1]{-p})=$ $K(\pi, \sqrt[p-1]{-p})=K\left(\pi, \zeta_{p}\right)$ is the splitting field of $f$ over $K$. (where $\zeta_{p}$ is a primitive $p$-th root of unity). Furthermore, since $X^{p-1}+p$ is Eisenstein, $K\left(\pi, \zeta_{p}\right) / K$ is totally ramified of degree $p(p-1)$ ( $K$ with no the $p$-th roots of unity), otherwise $L / K$ is normal. then:

Theorem 3.4. With the current notations, let $L / K$ be a separable extension of degree $p$. If $v_{L}\left(a_{i}\right) \geq v_{L}(p)+p=p(e+1)$ for $i, 1 \leq i \leq p-1$ then the splitting field of $f$ over $K$ is $K(\pi, \sqrt[p-1]{-p})=K\left(\pi, \zeta_{p}\right)$. Furthermore $K\left(\pi, \zeta_{p}\right) / K$ is totally ramified of degree $p(p-1),(K$ with no $p$-th roots of unity). Otherwise $K(\pi) / K$ is normal of degree $p$.

Now let us generate the intermediate extension another way:

Theorem 3.5. With the current notations, let $L / K$ be a separable extension of degree $p$. Then there exists $c \in K^{\star}$, unique up to $K^{\star(p-1)}$, such that the following hold:

- $L(\sqrt[p-1]{c})$ is the Galois closure of $L / K$

- For every $\tau \in \operatorname{Gal}(L(\sqrt[p-1]{c}) / K)$, and $\sigma \in \operatorname{Gal}(L(\sqrt[p-1]{c}) / K(\sqrt[p-1]{c}))$, we have $\tau \sigma \tau^{-1}=\sigma^{a}$, with $a=\frac{\tau(\sqrt[p-1]{c})}{\sqrt[p-1]{c}}$ modulo $p$.

Proof. $K$ contains the $p-1$-th roots of unity, $F / K$ is Kummer cyclic of degree $m$, so $F=$ $K(\sqrt[m]{b}), b \in K^{\star} . K(\sqrt[m]{b})=K(\sqrt[m]{d}) ;$ if and only if there exists an integer $k \geq 1 ;$ with $(k, m)=1$ 
such that $d \in b^{k} K^{\star m}$. Up to take $c=b^{(p-1) / m}, L F=L(\sqrt[p-1]{c})$.

Now $\tau(\sqrt[p-1]{c})=\sigma^{i}\left(\varepsilon^{j}(\sqrt[p-1]{c})\right)=\sigma^{i}\left(\zeta_{p-1}^{j} \sqrt[p-1]{c}\right)=\zeta_{p-1}^{j} \sqrt[p-1]{c}$, for every $\tau \in \operatorname{gal}(L(\sqrt[p-1]{c})=L F / K)$. So $\frac{\tau(\sqrt[p-1]{c})}{p-\sqrt[1]{c}}$ is a unit of $L / F$. $\zeta_{p-1}^{j}$ does not depend on $c$ but on the coclass $c K^{\star p-1}$ only. Indeed $\frac{\tau(\sqrt[p-1]{c})}{\sqrt[p-1]{c}}=\frac{\tau(\sqrt[p-1]{d})}{\sqrt[p-1]{d}}$, if and only if $\tau\left(\sqrt[p-1]{\frac{c}{d}}\right)=\sqrt[p-1]{\frac{c}{d}}$, that is $\frac{c}{d} \in K^{\star p-1}$.

Set $\theta=\sigma(\pi)-\pi$ so $\theta \equiv 0 \bmod \pi^{v+1}$ and $\pi_{1}=\tau(\pi)$ it is uniformizer too. So $\sigma\left(\pi_{1}\right)-\pi_{1}=$ $u(\sigma(\pi)-\pi)=u \theta$ with $u$ unit of $L F . u \equiv 1 \bmod \pi$, as $\sigma(\tau(\pi)-\pi) \equiv \tau(\pi)-\pi \bmod \pi$, so the class of $\frac{\tau(\theta)}{\theta} \bmod \pi$ is independent of $\pi$ and depends on $\tau$ and $\sigma$ only. Then write $\theta=\sigma \tau^{-1}\left(\pi_{1}\right)-\tau^{-1}\left(\pi_{1}\right)$, that is $\tau(\theta)=\tau \sigma \tau^{-1}\left(\pi_{1}\right)-\pi_{1}$. Now, since $\operatorname{gal}(L F / F)=<\sigma>$ is a normal subgroup of $\operatorname{gal}(L F / K)$ which is not abelian we have $\tau \sigma \tau^{-1}=\sigma^{a}$, with $1 \leq a \leq p-1$, therefore $\tau(\theta)=\left(\sigma^{a}\left(\pi_{1}\right)-\pi_{1}\right)$. Since the equality between ideals $\sigma\left(\left(\pi^{t}\right)\right)=\left(\pi^{t}\right)$ holds, by successive substitutions we get $\sigma^{a}\left(\pi_{1}\right)-\pi_{1} \equiv a\left(\sigma\left(\pi_{1}\right)-\pi_{1}\right) \equiv a(\sigma(\pi)-\pi)$

modulo $\pi^{v+2}$, that is $\tau(\theta) \equiv a \theta$ modulo $\pi^{v+2}$ for $1 \leq a \leq p-1$, finally we get

$$
\frac{\tau(\theta)}{\theta} \equiv a \text { modulo } \pi^{v+1} \quad \text { that is modulo } p \quad \text { for } \quad 1 \leq a \leq p-1
$$

From (1.9); $c=N_{L / K}(\gamma)=N_{L F / F}(\gamma) ; \gamma=-k a_{k} \pi^{k-1}, a_{k}$ is the principal coefficient of $f$. By (1.6) $\sqrt[p-1]{\gamma} \equiv \theta \bmod \pi \Rightarrow N_{L F / F}(\sqrt[p-1]{\gamma}) \equiv N_{L F / F}(\theta) \equiv \theta^{p} \equiv \theta \bmod \pi$, then finally

$$
\frac{\tau\left(N_{L F / F}(\sqrt[p-1]{\gamma})\right)}{N_{L F / F}(\sqrt[p-1]{\gamma})} \equiv a \text { modulo } \pi^{v+1} \quad \text { that is modulo } p \text { for } 1 \leq a \leq p-1
$$

Q.E.D.

\subsection{Explicit construction of the splitting field}

\subsubsection{Interpretation in case the principal coefficient is not $a_{p}$ :}

By a simple calculation we get the following Theorem ( 3.6) through the equality:

$$
\sqrt[p-1]{N_{L / K}(\gamma)}=\xi_{p-1} k a_{k}\left(-a_{0}\right)\left(\frac{v}{t}+1\right) \sqrt[p-1]{-k a_{k}\left(-a_{0}\right)^{-p v_{K}\left(a_{k}\right)}} .
$$

Theorem 3.6. With the current notations, let $L / K$ be a separable extension of degree $p$. If there exists an index $k, 1 \leq k \leq p-1$ such that $v_{L}\left(a_{k}\right)+k=\inf _{1 \leq i \leq p}\left(p v_{K}\left(a_{i}\right)+i\right)$ (hence necessarily $\left.v_{L}\left(a_{k}\right)+k<v_{L}(p)+p\right)$ then the splitting field of $f$ over $K$ is $K\left(\pi,\left(-a_{0}\right) \sqrt[v]{\frac{v}{t}} \sqrt[p-1]{-k a_{k}\left(-a_{0}\right)^{-p v_{K}\left(a_{k}\right)}}\right)$.

Remark 3.7. It is clear that if the condition ( 1.13) is satisfied then $K(\pi) / K$ is normal.

$$
\sqrt[p-1]{-k a_{k}\left(-a_{0}\right)^{-v_{K}\left(a_{k}\right)}} \in K(\pi)
$$

Particular case $k=1$.

Corollary 3.8. With the hypothesis and notations of theorem (3.3), if:

1. $v_{L}\left(a_{1}\right) \leq v_{K}\left(a_{i}\right)$ for every $i, 2 \leq i \leq p-1$ and 
2. $v_{L}\left(a_{1}\right) \leq v_{L}(p)$,

then the splitting field of $f$ over $K$ is $K\left(\pi, \sqrt[p-1]{-a_{1}}\right)$.

If $a_{1}=p \alpha_{1} ; \alpha_{1} \equiv 1 \bmod \mathfrak{P}_{K},(K$ a local number field) the splitting field of $f$ over $K$ is $K\left(\pi, \sqrt[p-1]{-a_{1}}\right)=K(\pi, \sqrt[p-1]{-p})=K\left(\pi, \xi_{p}\right)$, where $\xi_{p}$ is a primitive $p$-th root of unity.

Lemma 3.9. Let $(m, p)=1$ and $x \in K^{\star}$, then $K(\sqrt[n]{x}) / K$ is an unramified extension precisely if $x \in U_{K} K^{\star n}$. (See [11]Lemma 5.3.)

From Lemma (3.9) with $F=K\left(\left(-a_{0}\right)^{\frac{v}{t}} \sqrt[p-1]{-k a_{k}\left(-a_{0}\right)^{-p v_{K}\left(a_{k}\right)}}\right)$, we have:

Lemma 3.10. With the conditions of Theorem (3.3)

$(p-1)$ divides $\left(v_{K}\left(a_{k}\right)+k-1\right)$ (ie. the break is integer), if and only if $F / K$ is unramified.

\section{Generation by discriminant:}

We have $\Delta(f)=(-1)^{\frac{p(p-1)}{2}} N_{K(\pi) / K}\left(f^{\prime}(\pi)\right) \cdot f^{\prime}(\pi)=\sum_{i=1}^{p} i a_{i} \pi^{i-1}$

$=k a_{k} \pi^{k-1}\left(1+\sum_{i \neq k} r_{i} \pi^{i-1}\right)$, with $r_{i}$ suitable choosen integers. Then it is clear that $v_{L}\left(r_{i} \pi^{i-1}\right)>$ 0 , for every $i, 1 \leq i \leq p$ and $i \neq k$ and therefore, $\left(1+\sum_{i \neq k} r_{i} \pi^{i-1}\right) \in U_{L}^{1}$, thus $N_{0}=$ $N_{L / K}\left(1+\sum_{i \neq k} r_{i} \pi^{i-1}\right) \in U_{K}^{1}$, and then $\sqrt[p-1]{N_{0}}=N^{\prime} \in K$. Indeed, since $U_{K}^{1} \supseteq N_{L / K}\left(U_{L}^{1}\right)$ and if $L / K$ is normal and totally ramified $N_{L / K}\left(U_{L}^{1}\right)$ is a subgroup of index $p$ of $U_{K}^{1}$. Now $N_{L / K}\left(-f^{\prime}(\pi)\right)=$ $N_{L / K}\left(-k a_{k} \pi^{k-1}\right) \cdot N_{0}, \quad$ therefore $\sqrt[p-1]{-N_{L / K}\left(f^{\prime}(\pi)\right)}=\xi_{p-1} \sqrt[p-1]{N_{L / K}\left(-k a_{k} \pi^{k-1}\right)} \cdot N^{\prime}$, then $L\left(\sqrt[p-1]{-k a_{k} \pi^{k-1}}\right)=K\left(\pi, \sqrt[p-1]{-N_{L / K}\left(f^{\prime}(\pi)\right)}\right)=K\left(\pi, \sqrt[p-1]{(-1)^{\frac{p(p-1)}{2}+1} \Delta(f)}\right)$.

Theorem 3.11. With the conditions of Theorem (3.3). If there exists an index $k$, $1 \leq k \leq p-1$ such that $v_{L}\left(a_{k}\right)+k=\inf _{1 \leq i \leq p}\left(v_{L}\left(a_{i}\right)+i\right)$. Then $K\left(\sqrt[p-1]{(-1)^{\frac{p(p-1)}{2}+1} \Delta(f)}\right) / K$ is a cyclic Kummer extension of degree $m, m$ dividing $p-1$.

Furthermore, the splitting field of $f$ over $K$ is $K\left(\pi, \sqrt[p-1]{(-1)^{\frac{p(p-1)}{2}+1} \Delta(f)}\right)$.

\subsubsection{Interpretation in case the principal coefficient is $a_{p}$ :}

\section{Generation by discriminant:}

$f^{\prime}(\pi)=\sum_{i=1}^{p} i a_{i} \pi^{i-1}=p \pi^{p-1}\left(1+\sum_{i=1}^{p-1} r_{i} \pi^{i-1}\right)$ with $v_{L}\left(r_{i} \pi^{i-1}\right)>0$, for every $i$,

$1 \leq i \leq p-1$, so $N_{L / K}\left(-f^{\prime}(\pi)\right)=N_{L / K}\left(-p \pi^{p-1}\right) \cdot N_{0}=(-p)^{p}\left(-a_{0}\right)^{p-1} \cdot N_{0}$; that is $\sqrt[p-1]{-N_{L / K}\left(f^{\prime}(\pi)\right)}=p \zeta_{p-1} \sqrt[p-1]{-p} a_{0} N$, with $N \in K$ thus the splitting field is $K(\pi, \sqrt[p-1]{-p})=K\left(\pi, \zeta_{p}\right)=K\left(\pi, \sqrt[p-1]{(-1)^{\frac{p(p-1)}{2}+1} \Delta(f)}\right)$. With the current notations:

Theorem 3.12. $K$ being a finite extension of $\mathbb{Q}_{p}$. if $v_{L}\left(a_{i}\right)+i \geq v_{L}(p)+p=p(e+1)$ for every $i$ , $1 \leq i \leq p-1$ then $K(\pi) / K$ is normal if and only if the $p$-th roots of unity lay in $K$, otherwise the splitting field of $f$ over $K$ is $K\left(\pi, \zeta_{p}\right)=K\left(\pi, \sqrt[p-1]{(-1)^{\frac{p(p-1)}{2}+1} \Delta(f)}\right)$. 


\subsection{Completeness and generation}

The generation above by a $(p-1)$-th root of the discriminant in Propositions (3.11) and (3.12), was done in a local case with finite residue field, so the completeness is a necessary. Here, a counterexample of an Eisenstein polynomial defined on $\mathbb{Q}$ its splitting field can not be generated by a $(p-1)$-th root of the discriminant, even by adjoining the $(p-1)$-th roots of unity to $\mathbb{Q}$, and the splitting field has a solvable Galois group.

Example 3.13. (Counter-Example):

Consider the number $\alpha=\sqrt[5]{\sqrt{26}+5}-\sqrt[5]{\sqrt{26}-5}$.

By calculation of successive powers of $\alpha$ we get the minimal polynomial of $\alpha, \operatorname{Irr}(\alpha, \mathbb{Q})(X)=$ $X^{5}+5 X^{3}+5 X-10$ (Eisenstein), $\alpha$ the single real root, $(\mathbb{Q}(\alpha) \subset \mathbb{R})$. Set $r=\sqrt[5]{\sqrt{26}+5}$, so we have $\alpha=r-1 / r$, and $\alpha_{j}=r \zeta_{5}^{j}-1 / r \zeta_{5}^{j}$, ( $\zeta_{5}$ is a primitive 5 -th root of unity). By a similar calculation of successive powers of $\alpha_{j}$ we get that $\alpha_{j}$ and $\alpha$ are conjugate (same minimal polynomial). So $\operatorname{Irr}(\alpha, \mathbb{Q})(X)=\prod_{j=0}^{4}\left(X-\alpha_{j}\right)=\prod_{j=0}^{4}\left(X-\left(r \zeta_{5}^{j}-1 / r \zeta_{5}^{j}\right)\right)$.

\section{1-st case:}

Consider $K=\mathbb{Q}_{5} \cdot \operatorname{Irr}\left(\alpha, \mathbb{Q}_{5}\right)(X)=\operatorname{Irr}(\alpha, \mathbb{Q})(X)$ and is still Eisenstein, then with respect to (Theorem 4.1. page 336 in $[9]), \mathbb{Q}_{5}(\alpha) / \mathbb{Q}_{5}$ is not normal. According thz study above the splitting field $E$ of $\operatorname{Irr}\left(\alpha, \mathbb{Q}_{5}\right)$ over $\mathbb{Q}_{5}$ is of degree dividing 20 .

Now since none of the nonzero coefficients of $f$ is divisible by 25 the principal coefficient of $f$ is $a_{1}=5$ then thanks to corollary (3.8) and Theorem (3.11) the splitting field of $f$ over $\mathbb{Q}_{5}$ is $\mathbb{Q}_{5}\left(\alpha, \sqrt[4]{-a_{1}}\right)=$ $\mathbb{Q}_{5}\left(\alpha, \sqrt[4]{(-1)^{\frac{5(4)}{2}+1} \Delta(f)}\right)$. Furthermore, the discriminant of $\operatorname{Irr}\left(\alpha, \mathbb{Q}_{5}\right)$ is $\Delta(f)=338000000=$ $5^{5} .10816=5^{5} .16 .26^{2}$. As $10816 \equiv 1$ modulo 5 it is then a 4 -power in $\mathbb{Q}_{5}, \mathbb{Q}_{5}\left(\sqrt[4]{(-1)^{\frac{5(4)}{2}+1} \Delta(f)}\right)=$ $\mathbb{Q}_{5}(\sqrt[4]{-5})=\mathbb{Q}_{5}\left(\xi_{5}\right)$. That is $E=\mathbb{Q}_{5}\left(\alpha, \xi_{5}\right)$

\section{2-nd case:}

$K=\mathbb{Q}(i)$ (with $i^{2}=-1$ ). The discriminant of $\mathbb{Q}(i)$ is -4 , it is not divisible by 5 , it does not ramify in $\mathbb{Q}(i), \operatorname{Irr}(\alpha, \mathbb{Q})$ is still Eisenstein in $K$. The splitting field $M$ of $\operatorname{Irr}(\alpha, \mathbb{Q})$, over $\mathbb{Q}$ has a solvable group of degree 20 (Software Pari), explicitly $<\sigma^{5}=\tau^{4}=1, \tau^{-1} \sigma \tau=\sigma^{2}>$. $M$ is included in $\mathbb{Q}\left(r, \zeta_{5}\right) / \mathbb{Q}$ which is of degree at most $40\left(r\right.$ is a root of the polynomial $\left.X^{10}-10 X^{5}-1\right)$. Since, $r^{5}=5+\sqrt{26}$ then $\mathbb{Q}\left(r^{5}\right)=\mathbb{Q}(\sqrt{26}) . \mathbb{Q}\left(\alpha, \zeta_{5}, \sqrt{26}\right)=\mathbb{Q}\left(\alpha, \zeta_{5}, r^{5}\right)$ is included in $\mathbb{Q}\left(r, \zeta_{5}\right)$. Since $\mathbb{Q}\left(\alpha, \zeta_{5}, \sqrt{26}\right) / \mathbb{Q}$ is of degree 40 then $\mathbb{Q}\left(\alpha, \zeta_{5}, \sqrt{26}\right)=\mathbb{Q}\left(r, \zeta_{5}\right)$, and the splitting field $M$ is then included in it.

By degrees consideration $K(\sqrt[4]{-5}) \subset K\left(\sqrt{26}, \zeta_{5}\right)$. Ad absurdum assume that $\sqrt[4]{-5} \in K\left(\sqrt{26}, \zeta_{5}\right)=$ $\mathbb{Q}\left(\sqrt{26}, \zeta_{20}\right) . \mathbb{Q}\left(\sqrt{26}, \zeta_{20}\right) / \mathbb{Q}$ being abelian cannot contain the non-normal extension $\mathbb{Q}(\sqrt[4]{-5}) / \mathbb{Q}$, so $\sqrt[4]{-5} \sqrt{26}$ does not lay in $K\left(\alpha, \sqrt{26}, \zeta_{5}\right)$ neither to the splitting field of $\operatorname{Irr}(\alpha, K)=\operatorname{Irr}(\alpha, \mathbb{Q})$ over $K$ that is included in it. Then the counter example. 


\section{References}

[1] E. Artin, Galois Theory, Univ. of Notre Dame Press, Notre Dame, 1944, Second edition.

[2] M.Hazewinkel, Local class field theory is easy, Adv. in Math.18 (1975), 148-181.

[3] B. Huppert, Endliche Gruppen I, Grundlehren der mathematischen Wissenschaften Volume 134, 1967

[4] G. James And M. LiebeCK, Representations and characters of groups, Cambridge University Press, New York, 2001.

[5] M.Krasner, Sur la primitivite des corps p-adiques, Mathematica Cluj t:13 (1937) pp. 72-191.

[6] M. Krasner, Nombres des extensions de degre donne d'un corps p-adique, Les tendances geometriques en Algebre et en theorie des nombres, Edition du centre national de la recherche scientifique Paris (1966) pp. 143-169.

[7] S. Lang, Algebraic number theory, Addison-Wesley publishing company, INC, 1968.

[8] A. Lbekkouri, On the Ore-Krasner equation, Scientiae Mathematicae Japonicae Vol. 74, No. 2 and 3 Whole Number 268 December 2011 pp. 121-134.

[9] A. Lbekkouri, On the construction of normal wildly ramified extensions over $Q_{p} p \neq 2$, Archiv der Math.Volume 93, Number 4 (2009), 331-344.

[10] A. Lbekkouri, On the construction of normal wildly ramified extensions over $Q_{2}$, Archiv der Mathematik Volume 93, Number 3 (2009), 235-243

[11] J. Neukirch, Class Field Theory (Grundlehren DerMathWissenschaften, 1986).

[12] P. Ribenboim, L'Arithmetique des corps Volume 2, Hermann Paris 1972.

[13] L. Ribes and P.Zalesskii, Profinite groups, A series of Modern surveys in Mathematics, Volume 40 Springer 2000.

[14] P. Rotman, An introduction to group theory, Springer Graduate texts in Mathematics, 2010.

[15] I.R. Safarevič , On p-extensions, Amer. Math. Soc. Transl. 4(2) (1954).

[16] J.P. Serre, Une formule de masse pour les extensions totalement ramifiees de degre donne dun corps local, Comptes Rendus 286, 1978, pp. 1031-1036.

[17] J.P. Serre, Local fields, Springer 1979. 